\title{
Benetzung von TMP und CTMP aus Holz sowie gebrauchten mitteldichten Faserplatten (MDF)
}

\author{
E. Roffael $\cdot$ B. Dix $\cdot$ C. Behn $\cdot$ G. Bär
}

Published online: 13 Juli 2008

(c) The Author(s) 2008

\section{On the wettability of fibres made by the TMP- and CTMP-process from pine wood and recycling medium density fibreboards (MDF)}

Subject TMP (thermo-mechanical pulps) and CTMP (chemo-thermo-mechanical pulps) were made from pine wood and recycling MDF, which has been prepared from pine wood too. The fibres were tested for their wettability using the method developed by Roffael et al. (2002). The results reveal that TMP are much less wettable in comparison to CTMP from the same wood. Moreover, fibres made from

Förderung: Das Verbundvorhaben zwischen der Universität Göttingen, dem Fraunhofer-Institut für Holzforschung und dem Institut für Zellstoff und Papier der Papiertechnischen Stiftung wurde mit Mitteln des Bundesministeriums für Wirtschaft und Arbeit (BMWA) durch die Arbeitsgemeinschaft industrieller Forschungsvereinigungen (AiF-Nr. 14801 BG) über den Internationalen Verein für Technische Holzfragen (iVTH) gefördert.

\section{E. Roffael ( $\bullet)$}

Georg-August-Universität Göttingen,

Büsgenweg 4, 37077 Göttingen, Deutschland

e-mail: eroffae1@gwdg.de

B. Dix

Wilhelm-Klauditz-Institut (WKI),

Fraunhofer-Institut für Holzforschung,

Bienroder Weg 54 E, 38108 Braunschweig, Deutschland

e-mail: brigitte.dix@wki.fraunhofer.de

\section{Behn}

Büsgen-Institut,

Abteilung Molekulare Holzbiotechnologie

und Technische Mykologie,

Georg-August-Universität Göttingen,

Büsgenweg 2, 37077 Göttingen, Deutschland

G. Bär

Institut für Zellstoff und Papier (PTS-IZP),

Papiertechnische Stiftung (PTS),

Pirnaer Str. 37, 01809 Heidenau, Deutschland
UF-bonded MDF by the TMP- and CTMP-process are of much higher wettability compared to TMP and CTMP prepared directly from wood. No significant difference in the wettability between TMP and CTMP from UF-bonded MDF was detected. This behaviour has been attributed to the fact that the UF-resin in MDF degrades during thermohydrolytic pulping leading to the formation of ammonia and free urea. Therefore, pulping of UF-bonded boards takes place under chemo-thermo-mechanical conditions (CTMP).

\section{Material und Methodik}

Kiefernholzhackschnitzel wurden zum einen nach dem TMP-Verfahren bei $170^{\circ} \mathrm{C}$ für 5 Minuten aufgeschlossen und anschließend in einem Einscheibenrefiner zerfasert. Zum anderen wurden die Hackschnitzel nach dem CTMPVerfahren unter gleichen Bedingungen aufgeschlossen und zerfasert. Bei Anwendung des CTMP-Verfahrens erfolgte der Aufschluss in Anwesenheit von 0,25\% Natriumhydroxid (\% Feststoff bezogen auf Trockenmasse Holz). Zum Vergleich wurden Zerkleinerungsprodukte aus UF-Harzgebundenen MDF nach dem TMP-Verfahren (ohne Chemikalien) und CTMP-Verfahren $(0,15 \%$ Natriumhydroxid bezogen auf Trockenmasse MDF) unter den gleichen Bedingungen aufgeschlossen und zerfasert. Aus den TMP und CTMP wurden jeweils in Abmischung mit einem ungebleichten Kiefernsulfatzellstoff (Verhältnis $1: 1$ ) Prüfblätter nach der Zellcheming-Methode (Verein der Zellstoff- und Papier-Chemiker und -Ingenieure 1976) hergestellt. An den Prüfblättern wurde das Benetzungsverhalten gemäß der von Roffael et al. (2002) vorgeschlagenen Methode bestimmt. Hierbei wurde die Zeitspanne ermittelt, die ein Tropfen $(10 \mu l)$ einer Prüfflüssigkeit benötigt, um vollständig in das Prüfblatt einzudringen. 
Abb. 1 Benetzung in Sekunden (s) von Prüfblättern, hergestellt aus Abmischungen (Verhältnis $1: 1$ ) von TMP und CTMP aus Kiefernholz sowie UF-Harz-gebundenen MDF (Aufschlusstemperatur $170^{\circ} \mathrm{C}$ ) und Sulfatzellstoff, gemessen nach der Methode von Roffael et al. (2002)

Fig. 1 Wettability in seconds (s) of handsheets made from TMP and CTMP of pine wood as well as from UF-bonded MDF (pulping temperature $170^{\circ} \mathrm{C}$ ) and sulphate pulp (ratio $1: 1$ ), according to the method developed by Roffael et al. (2002)

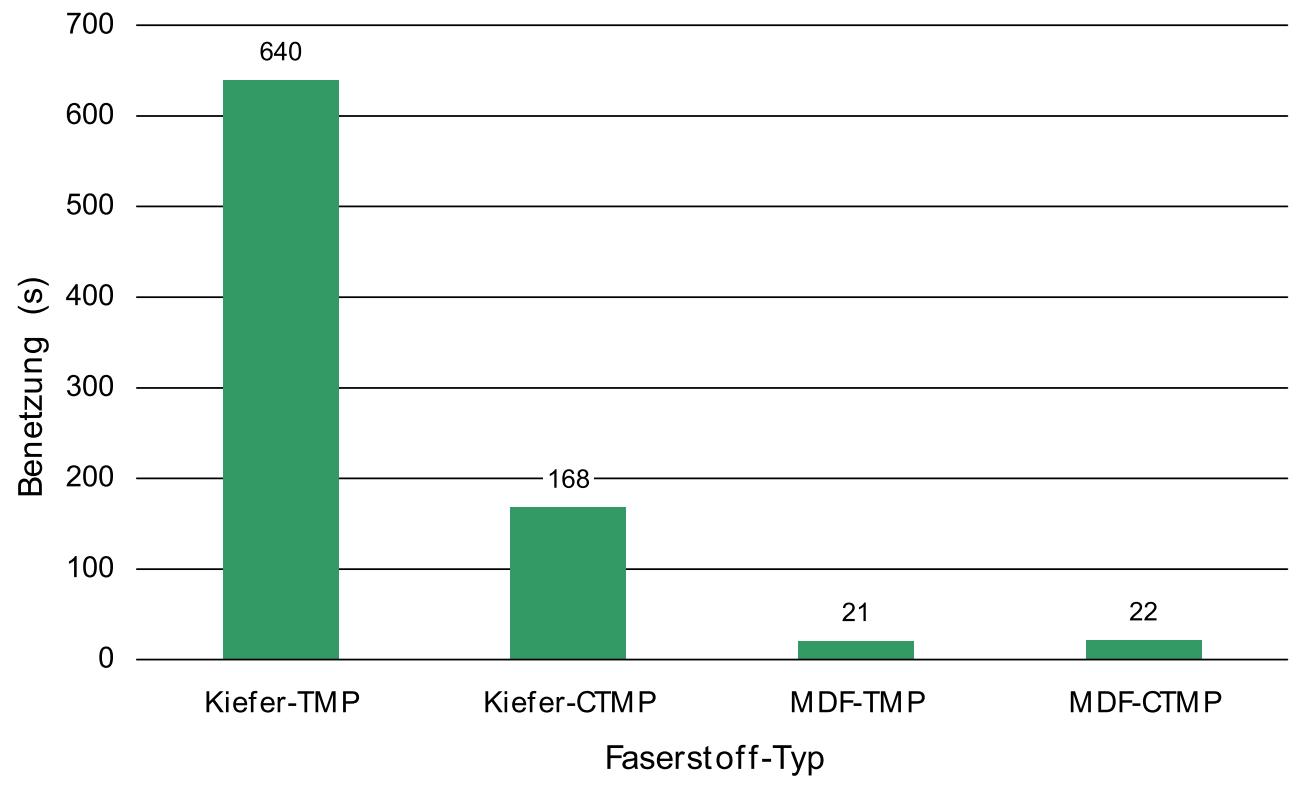

\section{Ergebnisse und Diskussion}

Die Ergebnisse der Messungen an Prüfblättern aus Refinerfaserstoffen in Kombination mit Sulfatzellstoff sind in Abb. 1 dargestellt. Daraus wird ersichtlich, dass die Prüfblätter mit TMP eine wesentlich schlechtere Benetzung als Prüfblätter mit CTMP aufweisen. Dies steht in Übereinstimmung mit den Ergebnissen von Schneider et al. (2007), wonach CTMP sich schneller benetzen lassen als TMP. Dies ist vermutlich zum einen auf den vergleichsweise höheren Gehalt an hydrophoben Extraktstoffen der TMP zurückzuführen und zum anderen auf das den CTMP anhaftende Alkali, das Extraktstoffe auflöst und die generelle Benetzbarkeit der Fasern mit wässrigen Lösungen verbessert.

Des Weiteren lassen die Ergebnisse erkennen, dass Prüfblätter mit TMP und CTMP aus UF-Harz-gebundenen MDF eine deutlich bessere Benetzung zeigen als Prüfblätter mit TMP und CTMP, die aus dem Kiefernholz direkt gewonnen wurden. Zwischen TMP und CTMP aus UF-Harz-gebundenen MDF bestehen hinsichtlich der Benetzung keine gesicherten Unterschiede. Dies beruht womöglich darauf, dass das UF-Harz in MDF während des Aufschlusses abgebaut wird und zur Bildung von Ammoniak und freiem Harnstoff führt. Demzufolge erfolgt der Aufschluss von UF-Harz-gebundenen MDF stets unter alkalischen Bedingungen, die auch beim CTMP-Verfahren vorliegen. Auf die Bildung von Ammoniak während der thermisch-hydrolytischen Behandlung von UF-Harz-gebundenen Spanplatten und MDF haben Franke und Roffael (1998) hingewiesen.

Open Access Dieser Artikel wird zu den Bedingungen der "Creative Commons Attribution Noncommercial License" zur Verfügung gestellt. Damit ist eine nichtkommerzielle Nutzung, Verbreitung und Vervielfältigung erlaubt, sofern die Autoren des Artikels und die genaue Quelle angegeben sind.

\section{Literatur}

Franke R, Roffael E (1998) Zum Recycling von Span- und MDFPlatten. Teil 2: Einfluss der thermischen Behandlung von zerkleinerten UF-gebundenen Span- und MDF-Platten sowie Kiefernspänen auf die Formaldehyd- und Ammoniakabgabe. Holz Roh- Werkst 56:381-385

Roffael E, Dix B, Schneider T (2002) Methode zur Erfassung der Benetzung von Holzfasern. Holz Roh- Werkst 60:347-348

Schneider T, Roffael E, Dix B, Erbreich M (2007) Zum Einfluss des Holzes sowie des Holzaufschlussverfahrens auf das Benetzungsverhalten von Kiefern- und Fichten-Faserstoffen. Holz Roh- Werkst 65:29-33

Verein der Zellstoff- und Papier-Chemiker und -Ingenieure (1976) Merkblatt V/8/76, Herstellung von Prüfblättern aus Halbstoffen mit Hilfe des Rapid-Köthen-Gerätes. Verein Zellcheming, Darmstadt 